\title{
PROCESSO DE DESIGN DE UM CHATBOT PARA PROGRAMA DE ENGAJAMENTO DO CLIENTE
}

\section{Chatbot Design Process for a customer engagement program}

\author{
Cecília V. Torres ${ }^{1}$, M.Sc Candidate \\ Jonysberg P. Quintino ${ }^{1}$, M.Sc \\ Frederico Oliveira $^{2}$, M.Sc. \\ Fabio Q. B. Silva ${ }^{1}$, D.Sc.
}

\author{
(1) UFPE \\ e-mail: cvtb@cin.ufpe.br \\ (2) UFPE \\ e-mail: jpq@cin.ufpe.br \\ (3) SIDI \\ e-mail: f.oliveira@samsung.com \\ (4) UFPE \\ e-mail: fabio@cin.ufpe.br
}

\begin{abstract}
Interfaces Conversacionais, Chatbots, Processo de Design
Chatbots viraram uma tendência na área de desenvolvimento de software nos últimos dois anos e com isso novos desafios para os designers de interação surgiram. Como um designer pode projetar uma interface que não é essencialmente gráfica? Esse artigo visa mostrar um possível caminho para designers projetarem chatbots.

Conversational Interface, Chatbots, Design Process

Chatbots have become a trend on software development field in the last two years and new challenges for interaction designers have emerged from it. How can we design an interface that is not essentially graphical? This article aims to show a possible way for designers to design chatbots.
\end{abstract}

\section{"Can machines think?"}

Turing, 1950

\section{Introdução}

A ideia de poder conversar com um computador fascina as pessoas há muito tempo e já foi tema de inúmeros livros e filmes de ficção científica. $\mathrm{O}$ considerado primeiro robô humanoide foi criado por Leonardo da Vinci em 1495, tinha o aspecto de um guerreiro, era capaz de ficar de pé, caminhar, abrir e fechar a boca e levantar os braços (Moran, 2006).

A partir do século XVIII, várias foram as tentativas de desenvolver máquinas capazes de imitar a fala humana até surgir a primeira máquina capaz de reconhecer os dígitos isolados, desenvolvida em 1952, nos laboratórios Bell (Rabiner et al., 1993). Desde então, a tecnologia de reconhecimento de fala progrediu rapidamente de um programa simples capaz de responder a um conjunto mínimo de palavras a sistemas altamente sofisticados como as interfaces conversacionais que existem hoje, conseguindo manter uma conversa fluida, como se estivesse sendo feita entre humanos.

Os assistentes virtuais são a prova disso. Eles existem para facilitar o cotidiano das pessoas, ajudando-as com tarefas que seriam feitas de forma muito mais custosa e muitas vezes desagradáveis. Essas interfaces já são extremamente populares nos dias de hoje. Os exemplos incluem o Siri, assistente virtual da Apple, o Google Assistant, da Google, o Cortana, da Microsoft, Alexa, da Amazon e Bixby da Samsung. Esses são exemplos de assistentes virtuais que respondem a comandos de voz.

Os chatbots são um tipo de assistente virtual que funciona por meio de uma interface de texto (muitas vezes também têm uma interface multimodal, podendo incluir fala e gestos). Eles vêm ganhando popularidade desde 2015 e os que utilizam inteligência artificial se tornaram uma tendência em 2016, especialmente com a integração com o messenger do Facebook e Slack, por exemplo, que 
permitem a criação de chatbots de forma muito mais rápida (Schlicht, 2016).

Muitos desses assistentes virtuais ajudam os usuários a executar várias tarefas em seus smartphones, como obter informações usando a pesquisa por voz ou por texto, encontrar restaurantes locais, obter rotas, definir o alarme, atualizar o calendário e participar de conversas gerais. Outros trazem características mais específicas, como monitoramento de exercícios físicos ou como preparar receitas e drinks. O termo interface conversacional é usado para referir-se à tecnologia que suporta a interação conversacional com os assistentes virtuais por meio de fala e outras modalidades de comunicação (Mc Tear et al., 2016).

O presente artigo apresenta o processo de design na construção de um chatbot para o XSolution (pseudônimo usado por questões comerciais e de sigilo) por meio da parceria do SIDI - Samsung Instituto de Desenvolvimento para Informática com o Projeto Samsung - Cin UFPE (Universidade Federal de Pernambuco). O objetivo do chatbot em questão é prover suporte para os usuários do $X$ Solution, um programa de capacitação e engajamento de vendedores, ajudando os usuários no suporte ao programa, regras e solução de problemas técnicos de forma rápida e eficaz. Por meio de metodologias de design para projetar artefatos digitais, esse artigo visa mostrar um possível caminho para designers projetarem chatbots, através de um guia de boas práticas.

\section{Fundamentação Teórica}

\subsection{Interfaces Conversacionais}

Interfaces conversacionais são softwares que permitem aos usuários interagirem com dispositivos usando fala ou linguagem natural como principal forma de comunicação. O termo interface conversacional foi cunhado por Hutchins (1989), onde ele as descreve como uma metáfora da conversação entre humanos, funcionando como um intermediário entre usuários e máquinas.

Algumas dessas interfaces podem usar modalidades adicionais de input e output, como caneta, toque, gestos e assim por diante, para aumentar a robustez do sistema e diminuir a carga cognitiva dos usuários. Elas envolvem, na interação entre usuário e interface, pares de sequência verbal como pergunta-resposta, solicitação-aceitação, sugestãorejeição e assim por diante.
A Figura 1 mostra os principais componentes de uma interface conversacional típica. O input é dado pelo usuário na forma de fala ou texto. Esse input é reconhecido e depois passado por um componente de compreensão da linguagem. O componente de compreensão faz uma representação de significado para o input dado. Se a entrada for executada em paralelo, as representações de significado parcial são geradas e fundidas na unidade de integração multimodal. Se as informações coletadas da representação de significado forem ambíguas, $\mathrm{o}$ sistema poderá solicitar esclarecimentos. A informação do discurso é mantida durante o processo, a fim de compreender um enunciado no contexto (Niculescu, 2011).

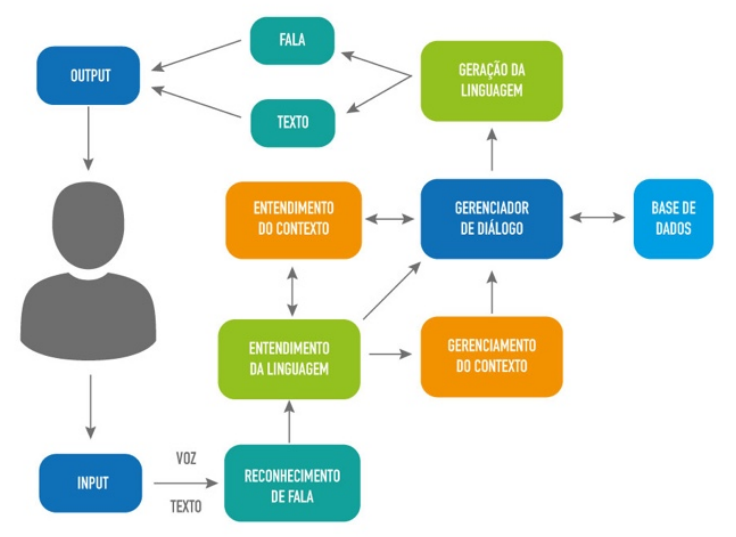

Figura 1 Arquitetura típica de uma interface conversacional. Fonte: Adaptado de Niculescu, 2011.

As interfaces conversacionais podem variar daquelas com estruturas de diálogo rudimentares, onde o computador tem o controle de interação completo, exigindo que o usuário responda a um conjunto de perguntas prescritas, até às interfaces de diálogo mais complexas, permitindo uma iniciativa de diálogo misto. Assim, interface conversacional é um termo global que pode se referir a uma interface de usuário de voz interativa, um sistema de diálogo falado, um sistema de resposta a perguntas multimodais ou um robô social usando a fala para se comunicar. (Niculescu, 2011)

\subsection{Origem do termo chatbot}

Os chatbots surgem a partir de um programa desenvolvido por Joseph Weizenbaum, em 1966, chamado ELIZA, que mimetiza uma terapeuta por meio de uma conversa muito convincente com seu paciente. É considerado o primeiro chatbot da história e inspirou toda uma geração de desenvolvedores desde então. (Mc Tear et al., 2016) 
"ELIZA simulava conversas por meio de textos préconfigurados para serem acionados por inputs de texto específicos. Se isso soa familiar é porque é a mesma estrutura que a maioria dos chatbots de hoje utilizam" (Muldowney, 2017). ELIZA ganhou bastante notoriedade por sua habilidade de "enganar" os usuários, fazendo-os acreditar que estavam tendo uma conversa real com outro humano. Além disso, foi considerada por alguns como o primeiro programa a passar no Teste de Turingi.

O termo chatbot tem origem no sistema CHATTERBOT, criado como um personagem para um jogo multi-user dungeon ${ }^{i i}$ (Mauldin, 1994). Sua principal tarefa era responder perguntas dos jogadores sobre a navegação através do calabouço, sobre outros jogadores e objetos disponíveis no mundo do jogo. O sistema simulava habilidades de conversação por meio de regras simples e alguns truques inovadores e "enganava" com sucesso os outros jogadores, fazendo-os crer que estavam conversando com outro usuário. (Klüwer, 2011).

Embora Weizenbaum não tenha chamado ELIZA de "chatbot", a origem do termo dado a este tipo de interface conversacional é geralmente assumida como começando com ELIZA, e o CHATTERBOT foi desenvolvido diretamente com base na experiência deste programa (Klüwer, 2011).

\subsection{Por que chatbots?}

Nos dias de hoje, usuários têm usado mais dispositivos móveis que computadores desktop e está sendo cada vez mais difícil impressioná-los e engajá-los na web. O ecossistema de aplicativos rapidamente saturou, sendo também cada vez mais caro e árduo competir dentro desse mercado.

Curiosamente, os aplicativos que permanecem nos smartphones dos usuários são os de mensagem, como Whatsapp, Facebook Messenger, Slack ou Telegram. Os usuários passam boa parte de seu tempo online nesse tipo de aplicação.

"Os bots vão causar uma perturbação na indústria de software da mesma forma que as revoluções da web e dos dispositivos móveis fizeram" (Shevat, 2017). A revolução que a web e os dispositivos móveis causaram mudaram a indústria de software e o comportamento do usuário, e essas mudanças tornam o terreno bastante propício para as empresas de software investirem nos chatbots (Figura 2).

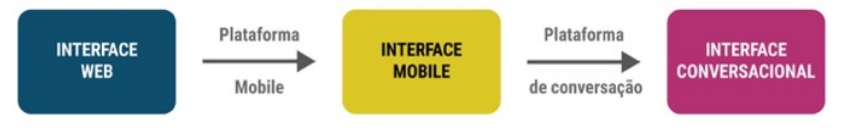

Figura $2 \mathrm{Da} W e b$ às interfaces conversacionais em dispositivos móveis. Adaptado de Shevat, 2017.

Chatbots são interfaces conversacionais que simulam conversas baseadas em texto. Uma regra fundamental para os designers de chatbots é que eles não precisam se parecer um ser humano. É importante ter uma comunicação fluida, consistente, mas é fundamental que o usuário saiba que ele está lidando com um chatbot e não com um humano.

Apesar da base de conversação dos chatbots ser textual, alguns elementos de interface têm sido usados para agilizar ou antecipar decisões dos usuários. Elementos gráficos como menus com tópicos que sugiram o que o usuário procura são exemplos desse tipo de recurso usado para facilitar o diálogo e agilizar a resolução do problema.

\section{Metodologia}

Projetar um chatbot é, acima de tudo, um processo de design centrado no usuário. Não se trata apenas do design de uma interface gráfica, mas do design de uma experiência. E essa experiência precisa ser a melhor possível.

"A experiência do usuário é uma consequência da apresentação, funcionalidade, desempenho do sistema, comportamento interativo e recursos de assistência de um sistema interativo, tanto de hardware quanto de software. É também uma consequência das experiências anteriores do usuário, atitudes, habilidades, hábitos e personalidade." (ISO 9241-11, 2010)

Para definir a experiência de uso do chatbot, dentro do contexto do problema em questão, fez-se necessário seguir alguns passos importantes no direcionamento da solução.

\subsection{Processo de Design do chatbot}

O processo de design do chatbot do XSolution teve como abordagem o Design Centrado no Usuário. $\mathrm{O}$ design centrado no usuário é "uma filosofia baseada nas suas necessidades e nos seus interesses, que dê atenção especial à questão de fazer produtos compreensíveis e facilmente utilizáveis" (Norman, 2006). 
"Todo o processo de design é tanto um processo criativo como um processo de solução de problemas" (Löbach, 2001). Como processo de solução de problemas, ele conta com 4 fases macro: análise do problema, geração de alternativas, avaliação das alternativas e realização da solução.

Esse processo é replicado em diversas metodologias de design, tal como a metodologia de Design Thinking, conforme mostra gráfico da Figura 3, que foca no conjunto de métodos e processos que propõe soluções com base na empatia e no foco nas pessoas afetadas pelo problema em questão, trazendo uma visão holística para a inovação.

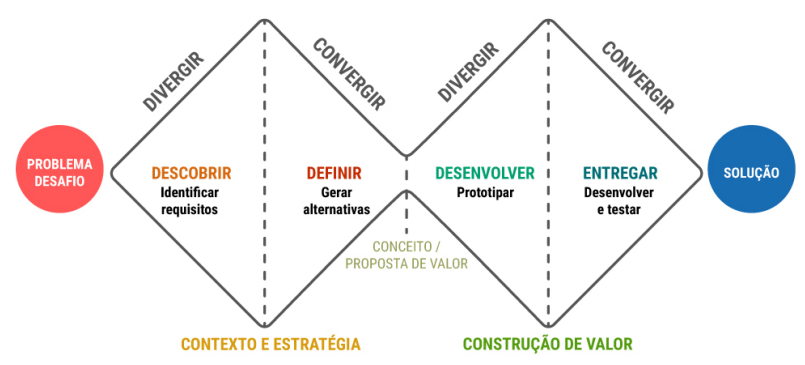

Figura 3 Processo de Design Thinking; modelo do Double Diamond

O modelo do Double Diamond propõe dois momentos: o momento de imersão no contexto do problema e o de construção de valor. A primeira fase é de interpretação e alinhamento dos achados para os objetivos do projeto, definição de princípios de design, propostas de interação e alternativas à solução. O segundo momento é da prototipação das ideias para desenvolvimento e validação da solução.

O primeiro passo para resolver um problema de design é o entendimento do problema, o que se está querendo sanar, que dores o usuário final tem. $\mathrm{O}$ segundo passo é conhecer bem esse usuário. Conhecer o usuário e o contexto de uso é fundamental para dar um passo inicial num projeto de chatbot. Sem essa etapa, o projeto pode estar fadado ao fracasso pois toda experiência é baseada em quem vai usufruir dela.

Em seguida, na fase de imersão, foi feita uma pesquisa dentro da temática dos chatbots, envolvendo também interfaces conversacionais em geral. Além disso, uma pesquisa de benchmark no contexto de chatbots de atendimento ao cliente.

$\mathrm{Na}$ fase de ideação e geração de alternativas foi quando se começou a pensar no chatbot propriamente dito. Personalidade, habilidades conversacionais, entre outros, foram definidas nessa fase do processo.

A última fase foi a de prototipação, que envolveu várias etapas, desde a criação dos fluxos

navegacionais, os primeiros rascunhos em papel, até a interface gráfica final, passando pelos wireframes, micro-interações e elementos gráficos de interação.

Assim, o chatbot fiou pronto para ser implementado pela equipe de desenvolvimento.

\subsection{Entendendo o problema}

Para começar a projetar o chatbot e suas interações, foi necessário entender o problema e conhecer o usuário que iria utilizá-lo.

O "Fale Conosco" do X Solution, um programa de relacionamento entre a Samsung e lojas parceiras, é um canal de contato dos vendedores.

O intuito do canal é o de tirar dúvidas e responder aos questionamentos dos usuários do programa de capacitação: questões sobre as regras do programa, suporte para problemas técnicos e até mesmo registro de reclamações.

O suporte ao cliente no "Fale Conosco" é realizado por um atendente por meio de mensagens de texto. Alguns questionamentos acabavam sendo repetitivos e a quantidade de dúvidas terminava por sobrecarregar o atendimento tanto na velocidade quanto na padronização das respostas.

Com intuito de auxiliar o "Fale Conosco" do $X$ Solution, foi solicitada a criação de um chatbot para melhorar e agilizar a comunicação entre usuários do programa de capacitação e a equipe de comunicação da Samsung, com objetivo de solucionar em tempo real os problemas do usuário do sistema de forma mais rápida, prática e assertiva, aumentando a satisfação do usuário final.

\subsection{Pesquisa e imersão}

Para projetar a experiência de uso do Chatbot, foi necessário fazer uma pesquisa do estado da arte dentro do tema de interfaces conversacionais, assistentes virtuais e chatbots, considerando o contexto de design de interação. Além da pesquisa na literatura, também foi feita uma pesquisa inicial de mercado por meio de um benchmark de soluções similares. 
"Um chatbot de domínio específico dá visibilidade a um único serviço, produto ou marca. Ele representa aquele serviço e os usuários associam o bot àquele serviço." (Shevat, 2017). Interfaces conversacionais para Serviço de Atendimento ao Consumidor (SAC) são aplicações já consolidadas no mercado. Como exemplos bem sucedidos de chatbots criados para $\mathrm{SAC}$, podemos citar a $\mathrm{Lu}$, assistente virtual do Magazine Luiza e o Bahianinho, assistente virtual das Casas Bahia, além de Anna, chatbot da IKEA, loja sueca de móveis, que foi um dos primeiros chatbots do tipo, criada em 2005. Este tipo de assistente, além de agilizar a comunicação e a assertividade na solução de dúvidas do usuário também é capaz de aprender com os diálogos, enriquecer seu vocabulário e ficar mais inteligente.

Shevat (2017) define alguns aspectos, habilidades e componentes que devem ser considerados na construção da interação com um chatbot: personalidade, avatar, nome e intervenção humana.

Personalidade: é a chave dos atributos que irão diferenciar um bot dos demais que oferecem um serviço similar. Fazendo uma analogia, seria como a escolha cromática dentro de uma interface gráfica de um aplicativo, algo que vai dar consistência em toda a interação e indicar aos usuários com que tipo de bot ele está lidando. Para a criação da personalidade, é importante considerar sempre o público-alvo e o contexto em que o usuário irá utilizar o chatbot. A personalidade do bot deve estar de acordo ao nível de descontração ou conservadorismo que o ambiente possui.

Avatar: o avatar é a representação projetada do usuário ou personagem dentro do ambiente imersivo, podendo ser um jogo ou mesmo um aplicativo. No caso do chatbot, provê-lo nome e face é uma das chaves para seu sucesso. Dotá-lo de personalidade é importante, mas é fundamental que o avatar também reflita essa qualidade. $O$ avatar também ajuda o usuário a identificar o chatbot $\mathrm{e}$ reconhecer com quem está falando.

Intervenção humana: sugerir correções no curso da conversa ou lidar com erros é uma estratégia inicial para um chatbot gerenciar as conversas. Caso ele não consiga, é necessário que o problema seja encaminhado a um humano.

\subsection{Ideação}

Nesta fase começou-se a pensar nas características que teria o chatbot. Qual sua personalidade? Que tipo de linguagem ele irá utilizar?

Para começar a projetar a interação do chatbot, foi necessário definir uma Persona (Figura 4). Por meio dela, foi mais fácil definir uma personalidade e o tipo de linguagem utilizada, que futuramente ajudaria na formulação dos diálogos nas mais diversas situações.

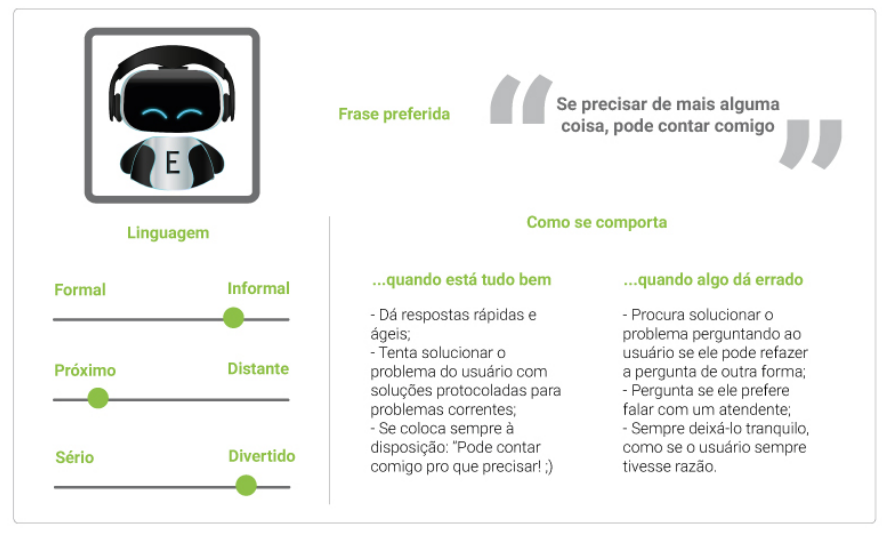

Figura 4 Persona do chatbot

Para a criação do avatar, foi identificado na pesquisa que a maioria dos bots possui uma fisionomia humana, caricaturizada ou mais realista, com intuito de humanizar o chatbot. Dotá-los de características humanas pode gerar mais empatia com o usuário.

No caso do chatbot do XSolution, o cliente escolheu algo que pudesse refletir os produtos da Samsung. Aspectos como modernidade, tecnologia, design de produto foram levados em consideração na hora do briefing. Nas reuniões de ideação para a criação do personagem chegou-se à conclusão que a ilustração deveria ser a de um robô simpático que, além de parecer um assistente virtual semelhante aos que existem no mercado, possuísse elementos estéticos de produtos Samsung. A conclusão é que essa seria a solução mais adequada e poderia se diferenciar do que é praticado comumente no mercado.

Definidos a personalidade e o avatar, foi necessário idealizar o tipo de linguagem que teria o chatbot. A interação e a comunicação também deve ser feita de forma natural, de modo que o usuário se sinta confortável como se estivesse falando com um atendente de verdade.

\subsubsection{Habilidades conversacionais}

O passo seguinte foi pensar nas habilidades conversacionais que o chatbot precisa ter para garantir a eficácia da sua interação com os usuários. 
Algumas habilidades conversacionais definidas para o XSolution são:

- Empatia: Mesmo para os atendentes de call-center, empatia é uma habilidade que poucos dominam. Se uma conversa é iniciada com muitas emoções negativas e críticas, normalmente as pessoas respondem com defesa e negatividade. Porém, no que diz respeito a atendimento ao cliente, é fundamental empatizar com os usuários, mesmo quando esses parecem estar errados.

- Oferecer opções: É importante deixar claro para o usuário o que o chatbot é capaz de fazer. Ao invés de dizer "O que eu posso fazer por você hoje", oferecer opções no início da interação pode ser muito mais eficaz.

- Lembrar de detalhes pessoais: Lembrar sempre o nome do usuário e o que ele acabou de perguntar é parte fundamental da fluidez da conversa.

- Não repetir perguntas/respostas: Ao invés de repetir uma pergunta ou resposta inúmeras vezes, uma maneira melhor de lidar com os erros dos usuários é oferecer uma solução alternativa. Se o usuário repete uma mesma pergunta geralmente significa que essa resposta não foi satisfatória.

Ao ter acesso a alguns diálogos reais entre o "Fale Conosco" e os usuários, foi possível mapear que tipos de questionamentos e problemas são mais comuns, quais os caminhos que a conversa pode seguir e assim projetar os fluxos de navegação com os cenários possíveis de comunicação e interação.

\subsection{Prototipação}

Chatbots podem ser criados baseados em regras ou por inteligência artificial, "incluindo deep learning, processamento de linguagem natural (NLP) e algoritmos de machine learning, e exigem grandes quantidades de dados" (Rouse, 2017). Chatbots alimentados por regras são mais limitados e somente respondem a comandos específicos e seguem fluxos de navegação bem definidos. Se o usuário diz algo errado, o sistema não saberá interpretar.

Já com os chatbots criados por meio de Inteligência Artificial, o usuário não precisa ser extremamente específico no que está dizendo, pois o bot aprende e entende linguagem natural, não apenas comandos. Com o passar do tempo, o chatbot aprende com as conversas e fica mais inteligente.
O chatbot do XSolution foi criado baseado em regras bem definidas. Ele tem um espectro limitado até onde pode ir e foi feito baseado nas possibilidades de interação que o usuário pode ter com o bot.

Para auxiliar na criação dos fluxos navegacionais, de interação e facilitar a criação dos diálogos, foi feita uma ontologia (Figura 5). De acordo com Castro et al. (2017) uma ontologia é uma representação de dados baseada em conceitos e seus relacionamentos, representada em um formato que pode ser lido tanto por seres humanos quanto por máquinas, detalhando seus principais conceitos e os relacionamentos entre si. A adoção de ontologias promove a gestão do conhecimento, desempenho, escalabilidade e reutilização. De acordo com AbdulKader e Woods (2015), um chatbot complexo é tão bom quanto sua base de conhecimento.

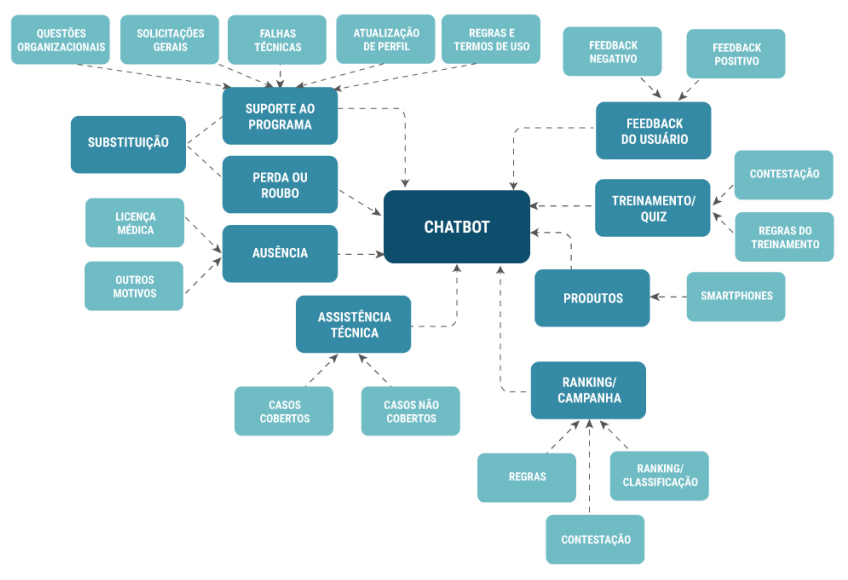

Figura 5 Fragmento da Ontologia do Chatbot. Adaptado de Castro et al., 2017.

A partir da ontologia, foi possível definir os fluxos de navegação que iriam dar suporte aos primeiros wireframes. O fluxo foi dividido em categorias (dúvidas, elogios, reposição, suporte, problemas técnicos, etc) e cada uma possuía um caminho específico. 


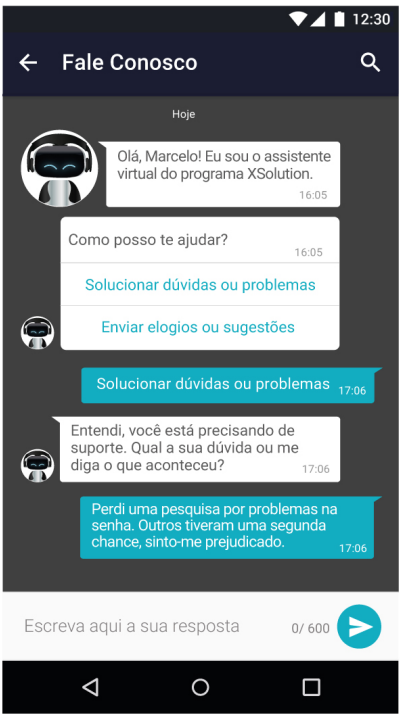

Figura 6 Tela inicial do chatbot com exemplo de conversa com usuário

A partir de uma ação do usuário, a conversa é inciada pelo chatbot, que dá as boas vindas, informa ao usuário o que faz e como pode ajudá-lo. Em seguida, são dadas opções ao usuário por meio de um pequeno menu que oferece as duas opções macro do menu: "solucionar dúvidas ou problemas" e "enviar elogios ou sugestões". Essa escolha foi feita baseada na maioria das questões dos usuário resolvidas pelo "Fale Conosco".

Apesar da interação do usuário com o chatbot ser textual, alguns outros elementos de interação foram utilizados com intuito de antecipar decisões do usuário e agilizar o atendimento.

Um menu carrossel surge sempre que o chatbot não consegue resolver às dúvidas do usuário por meio de texto. Esse menu é exibido ao usuário em formato de cards com as categorias disponíveis e oferece opções para o usuário solucionar seu problema ou dúvida. Ao clicar na opção desejada, a opção é repetida na caixa de diálogo logo abaixo do menu.

Caso o usuário não consiga resolver seu problema através do menu, existe a possibilidade de contato com um humano, através do envio de mensagem, ou fazer uma chamada telefônica por meio do chatbot, escolhendo a opção no card conforme mostra a Figura 7. Além disso, mesmo com as opções do menu, o usuário pode também escrever seu problema no campo de texto para que o bot possa novamente tentar uma resposta.

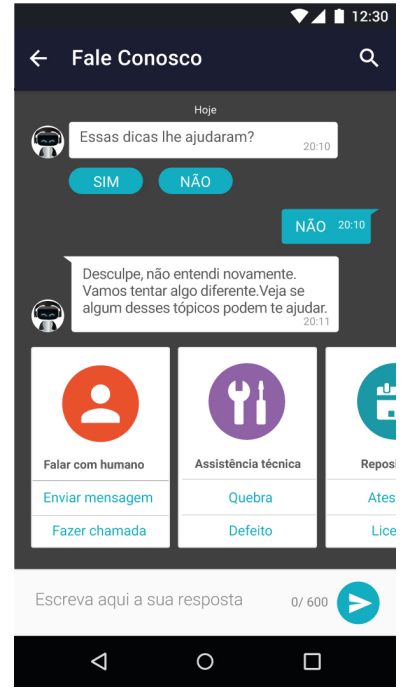

Figura 7 Tela com cards ofertando ao usuário atalhos às demais soluções para seus problemas

Além de responder às dúvidas dos usuários, $\mathrm{o}$ chatbot também tem a função de colher feedback dos usuários através de pequenas pesquisas de satisfação. São pesquisas para, além de medir o nível de satisfação do usuário com o programa, identificar os motivos para um mau desempenho num quiz ou saber o porquê do usuário não gostar de determinados tipos de conteúdo.

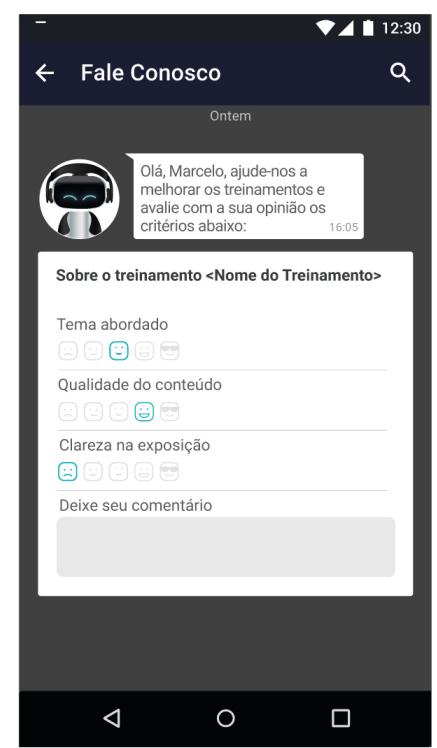

Figura 8 Tela com pequena pesquisa de feedback

Essas pesquisas são feitas no chatbot do XSolution por meio de questões respondidas rapidamente por meio de uma escala Likert ${ }^{\text {iii }}$. Esse é outro elemento de interação utilizado no chatbot que foge ao padrão de texto e dá uma função distinta à função original de tirar dúvidas e resolver problemas, mas também de colher feedback a respeito do programa. 


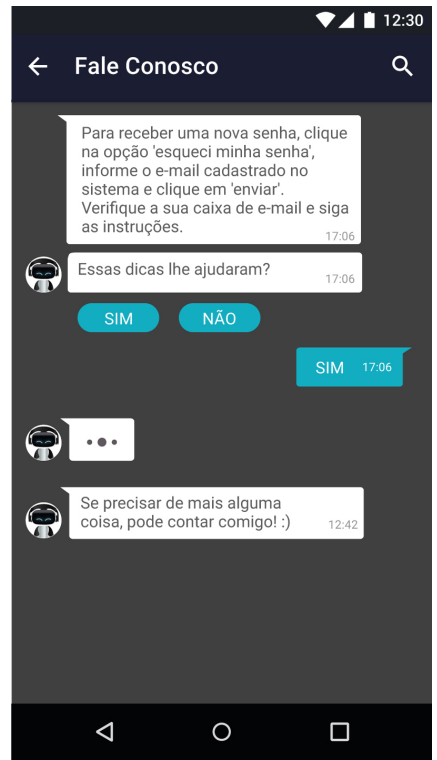

Figura 9 Tela com loading de mensagem do chatbot

Por fim, o chatbot do XSolution segue a abordagem de fornecer sempre feedback ao usuário, ou seja, não deixá-lo esperando por uma resposta sem saber o que está acontecendo. Quando uma mensagem estiver sendo gerada pelo chatbot, um feedback visual deve ser mostrado no diálogo, conforme visto na Figura 9.

\section{Resultados e Discussão}

Após alguns meses da implantação do chatbot no Fale Conosco do $X$ Solution, foram analisados os relatórios de atendimento ao cliente. No gráfico abaixo, pode-se ter uma amostra da relação de atendimentos realizados pelo chatbot em relação aos realizados pelos atendentes.

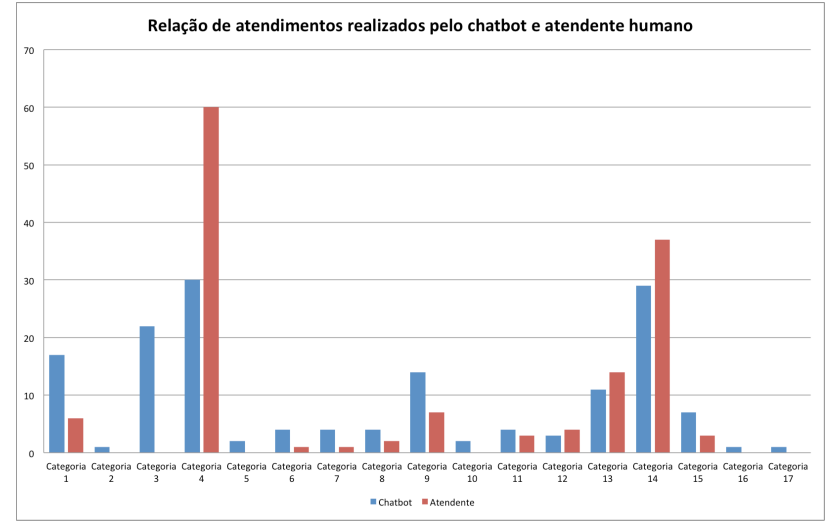

Figura 10 Gráfico comparativo da relação de atendimentos realizados

No período compreendido entre $1^{\circ}$ de março a 29 de Setembro de 2018, um total de 306 atendimentos foram realizados, dos quais 162 foram respondidos pelo chatbot e 138 pelos atendentes humanos. Dentro da Ontologia definida, das dezessete subcategorias atendidas pelo Fale Conosco, sete ( $41,1 \%$ do total) foram $100 \%$ atendidas pelo chatbot e treze $(76,48 \%$ do total) com percentual maior que $\mathrm{o}$ atendimento humano. Nas categorias em que o atendimento humano superou o do chatbot, o tipo de situação a ser tratado exigia a intervenção humana.

Observa-se, portanto, que dentro do escopo definido pela ontologia para o chatbot, constatou-se que ele foi mais efetivo que o atendimento humano.

Costuma-se dizer que design é uma forma de diálogo entre designer e usuário. Para entender a necessidade do usuário, conversamos com eles sobre suas dores e seus problemas. Discutimos com nossos colegas sobre propostas de soluções. É uma forma de colocar pra fora nossos pensamentos de forma clara e objetiva para que o outro os entenda.

No entanto, a conversação se tornou uma realidade cada vez mais relevante para o mundo dos designers no momento em que computadores começaram a se comunicar conosco. Conversa tornou-se uma interface de usuário. E como esse é nosso métier, é preciso estarmos preparados para projetarmos essas interfaces.

Por fim, seguem alguns aspectos importantes e de boas práticas para um projeto de chatbot:

- Entenda o problema do cliente: o que ele está querendo resolver? Quais as dores do seu usuário? Esse primeiro passo é fundamental para identificar se o chatbot é ou não uma solução para o problema.

- Conheça quem vai usar seu chatbot: usuário e contexto de uso são informações essenciais para qualquer projeto de design e não seria diferente para um chatbot. A experiência depende de quem vai fazer uso dela.

- Mergulhe no tema: pesquise soluções similares para problemas semelhantes ao que pretende resolver. Pesquise chatbots para problemas similares, o que fazem, como são e como agem.

\section{- Crie uma persona para o seu chatbot: criar} nome e avatar para seu bot é a melhor forma de criar familiaridade com ele. Além disso, ajuda a definir sua personalidade, tipo de linguagem usada, dentre outras características próprias. 
- Crie um chatbot empático: colocar-se no lugar do outro é uma virtude para poucos. Em atendimento ao cliente, quando muitas vezes ele está emocionalmente abalado com seus problemas e sem conseguir resolvê-los, empatia é uma habilidade fundamental. Dotar seu chatbot de empatia, reconhecer no diálogo do usuário quando ele pode estar irritado, é muito importante para saber como responder com empatia e paciência.

- Não tente enganar seu usuário: um chatbot não é uma pessoa e fazer o usuário pensar isso é um erro que um designer não deve cometer. Deixar a conversa fluida como se fosse uma conversa com um humano é diferente de querer fazer o usuário pensar que não está falando com uma máquina.

- Desenhe os fluxos de interação: eles vão ajudar a entender os caminhos que uma conversa pode seguir, auxiliar na tomada de decisão quanto a elementos de interação e quando o chatbot não irá conseguir resolver o problema do usuário. Se possível, guie o usuário num fluxo com a menor quantidade de interações possível para que ele chegue ao seu objetivo de forma mais rápida.

- Textos curtos: os diálogos devem ser quebrados em respostas curtas para não cansar o usuário e deixar a conversa fluida.

- Feedback: não deixe o usuário sem feedback. Quando o chatbot estiver processando alguma informação, um feedback visual precisa aparecer para o usuário entender o que está acontecendo. $\mathrm{O}$ contrário também é importante: solicitar que o usuário forneça informações sobre o atendimento, se ele foi útil ou eficaz, se resolveu seu problema.

- Elementos de interação: além de textos, o designer pode prover ao usuário uma interface com elementos de interação que antecipem decisões do usuário ou ajudem a deixar a interação mais fácil e rápida. Menus, botões com possíveis respostas para o usuário quando as questões tiverem respostas curtas, como "sim" ou "não", por exemplo, são alguns elementos que podem ser usados para facilitar e melhorar a experiência de uso.

- Intervenção humana: em casos em que o bot não consegue resolver o problema, avaliar as possibilidades de redirecionamento da conversa para um humano.

\section{Conclusões}

Por que chatbots são uma grande oportunidade de negócio hoje em dia? Porque chatbots são sistemas de conversação via texto e as pessoas estão cada vez mais usando aplicativos de mensagem instantânea ao invés de redes sociais, sites e outros aplicativos.

Os chatbots estão sendo usados cada vez mais em áreas como educação, recuperação de informações, negócios e comércio eletrônico, por exemplo, como assistentes on-line automatizados para complementar ou até mesmo substituir o serviço prestado por humanos em atendimento ao cliente.

Mesmo com a popularização dos chatbots nos últimos dois anos e com o surgimento de diversas plataformas que facilitam a construção de um chatbot, ainda há muitos desafios de design e desenvolvimento a serem encarados no futuro. Melhoria nas tecnologias para implementação, aumento da inteligência por trás do bot, fluxos de interação de chatbot guiados pelo contexto de negócios são alguns pontos que devem ser considerados para garantir uma boa experiência de uso.

"A dificuldade em projetar um chatbot é menos técnica e mais uma questão de experiência do usuário. Os chatbots mais bem-sucedidos serão aqueles em que os usuários irão querer voltar regularmente e os que fornecem valor consistente" (Hartman, em Schlicht, 2016)

O propósito deste paper foi mostrar um possível caminho para que designers de interação possam projetar seus próprios chatbots. $\mathrm{O}$ processo de design foi aqui descrito em suas etapas de forma detalhada, que caminhos o designer precisa seguir antes de começar a pensar nas interações. Foram apresentadas formas de interação além de campos de texto e balões de diálogo. Para além da interface gráfica, existem aspectos que precisam ser pensados por designers de interação, pois são eles que estão projetando para pessoas.

Com o guia de boas práticas apresentado ao final do artigo, é possível seguir um caminho dentro desses projetos de forma que o resultado seja um chatbot conciso, simples e fluido. Contando com uma boa tecnologia no desenvolvimento e testes com usuários ao final do projeto, o design de um chatbot torna-o a chave de seu sucesso. Quanto mais pesquisas com os usuários o designer fizer antes do desenvolvimento do chatbot menores serão os riscos 
e os custos para melhorar o desempenho do chatbot em um futuro próximo.

\section{Referências Bibliográficas}

ABDUL-KADER, A., WOOD, J. Survey on Chatbot Design Techniques in Speech

Conversation Systems. In (IJACSA) International Journal of Advanced Computer Science and Applications, Vol 6, N. 7, pp. 72-80 (2015)

CASTRO, Fernanda, TEDESCO, Patrícia; ALVES, Havana, QUINTINO, J., STEFFEN, J., OLIVEIRA, F., SOARES, F., SANTOS, A.L.M, SILVA, F.Q.B. Developing a Corporate Chatbot for a Customer Engagement Program: a Roadmap. 2017.

EPSTEIN, Robert, ROBERTS, Roberts, BEBER, Grace (Editors). Parsing the Turing Test:

Philosophical and Methodological Issues in the Quest for the Thinking Computer. Springer: New York, 2009

HUTCHINS, E. Metaphors for interface design. Em M.M. Taylor, F. Neel e D.G. Bouwhuis, editors, The Structure of Multimodal Dialogue. Elsevier Science Publishers, Nova York, 1989.

ISO. ISO 9241-210:2010. Ergonomics of humansystem interaction. Part 210: Human-centred design for interactive systems, 2010.

KLÜWER, Tina. From chatbots to dialog systems. Perez-Marin D, Pascual-Nieto I

(Editores). Conversational agents and natural language interaction: techniques and effective practices. IGI Global Publishing Group, Hershey, Pennsylvania, pp 1-22, 2011.

LÖBACH, Bernd. Design Industrial: Bases para a configuração dos produtos industriais. Tradução Freddy Van Camp. Edgard Blücher, Rio de Janeiro, 2001.

MC TEAR, Michael; CALLEJAS, Zoraida; GRIOL, David. The Conversational Interface: Talking to Smart Devices. Switzerland: Springer International Publishing Switzerland, 2016.

MORAN, Michael E. Epochs in Endourology The da Vinci Robot. Journal of endourology. Volume 20, N. 12, 2006
MULDOWNEY, Oisin. Chatbots: An

Introduction and Easy Guide to Make Your

Own. Dublin: Curses \& Magic, 2017.

NORMAN, Donald A. O Design do Dia a Dia. Rio de Janeiro: Rocco, 2006.

NICULESCU, Andreea Ioana. Conversational interfaces for task-oriented spoken dialogues: design aspects inuencing interaction quality. PHD Thesis. Holanda: University of Twente, 2011.

RABINER, L; JUANG, B-H. Fundamentals of speech recognition. Prentice Hall, Upper Saddle River, 1998.

ROUSE, Margaret. Chatbot. Atualizado em Novembro de 2017.

https://searchcrm.techtarget.com/definition/chatbot Acesso em 14 de Junho de 2018

ROUSE, Margaret. IMbot. Atualizado em Setembro de 2005.

https://searchdomino.techtarget.com/definition/IMbot. Acesso em 14 de Junho de 2018

SCHLICHT, Matt. The Complete Beginner's Guide To Chatbots: Everything you need to know. 2016

https://chatbotsmagazine.com/the-completebeginner-s-guide-to-chatbots-8280b7b906ca. Acesso em 26 de Junho de 2018.

SHEVAT, Amir. Design Bots: Creating Conversational Experiences. O'Reilly, 2017.

TURING, Alan M. Computing Machinery and Intelligence. Mind, Volume LIX, Issue 236, Páginas 433-460. Oxford, 1950.

\section{Agradecimentos}

Os resultados apresentados neste trabalho foram desenvolvidos como parte de um projeto colaborativo entre o Samsung Instituto de Desenvolvimento para Informática (SIDI) e o Centro de Informática da Universidade Federal de Pernambuco (CIn - UFPE), financiado pela Samsung Eletronica da Amazônia. Ltda., sob os auspícios da Lei Federal de Informática Federal no. 8248 / 91. Os autores gostariam de agradecer o apoio recebido da equipe da Samsung - SIDI, em especial a Juliana Steffen e Fabiana Florentin, pela parceria e pelo ambiente criativo e amigável que 
proporciona, e da UFPE, por ser uma instituição de fomento à pesquisa, ao desenvolvimento e à inovação. O professor Fabio Q. B. da Silva possui bolsa de pesquisa do Conselho Nacional de Pesquisa (CNPq), processo n 314523 / 2009-0.

\footnotetext{
${ }^{\mathrm{i}} \mathrm{O}$ Teste de Turing é uma avaliação criada por Alan Turing, conhecido como o pai da computação. Desenvolvido em 1950, o teste consiste em observar a capacidade de uma máquina ou software em exibir um comportamento inteligente, equivalente ao de um ser humano. O teste é utilizado por diversos sistemas que utilizam inteligência artificial e faz parte do conceito fundamental da filosofia da AI (Artificial Intelligence).

ii Multi-User Dungeon, ou MUD, é um RPG para múltiplos jogadores jogado online através de BBS ou Internet.

iii Escala Likert é um tipo de escala de resposta usada habitualmente em questionários e em pesquisas de opinião, onde os usuários especificam seu nível de concordância ou discordância com uma afirmação.
} 